\title{
Contribution à l'étude de la désinfection de l'eau par photosensibilisation avec des extraits de plantes
}

\author{
Teddy Makuba SUNDA*, Nicolas Kalulu Muzele TABA et Papy Mola MBALA \\ Université de Kinshasa, Faculté des Sciences, Département de chimie, B.P. 190, Kinshasa XI, République \\ Démocratique du Congo. \\ *Auteur correspondant ; E-mail : teddysunda@gmail.com
}

\section{RESUME}

Certaines plantes utilisées dans la pharmacopée traditionnelle pour soigner les infections microbiennes et parasitaires sont capables de générer l'oxygène singulet. Celui-ci est mis à profit pour désinfecter l'eau. L'étude de la désinfection de l'eau par photosensibilisation avec les extraits flavonoidiques de Aframomum alboviolaceum montre une inhibition complète des coliformes fécaux présents dans l'eau après une heure d'ensoleillement pour les deux produits isolés, par chromatographie sur colonne, des racines de cette plante. En ce qui concerne les échantillons d'eau traités avec ces deux produits et gardés à l'obscurité, aucune inhibition n'a été notée du début à la fin des expériences. Ceci laisse supposer que ces produits sont photosensibilisateurs et, qu'en présence du soleil, ceux-ci subissent une photoexcitation qui conduirait à la formation d'oxygène singulet. Ce dernier, une fois généré, détruit les coliformes fécaux présents dans l'eau.

(C) 2017 International Formulae Group. All rights reserved.

Mots clés: Photosensibilisation, Flavonoïdes, Aframomum alboviolaceum, oxygène singulet, temps d'ensoleillement.

\section{Contribution to the study of water disinfection by photosensitization with plant extracts}

\begin{abstract}
Some plants used in the traditional pharmacopoeia to treat microbial and parasitic infections are able to generate singlet oxygen. This is used to disinfect the water. The study of the disinfection of water by photosensitization with the flavonoid extracts of Aframomum alboviolaceum shows a complete inhibition of fecal coliforms present in the water after one hour of sunshine for the two isolated products by column chromatography of the roots of this plant. Regarding the water samples treated with these two products and kept in the dark, no inhibition was noted from the beginning to the end of the experiments. This suggests that these products are photosensitizers and that in the presence of the sun they undergo photoexcitation which would lead to the formation of singlet oxygen. The latter, once generated, destroys the fecal coliforms present in water.

(C) 2017 International Formulae Group. All rights reserved.
\end{abstract}

Keywords: Photosensitization, Flavonoids, Aframomum alboviolaceum, Singlet oxygen, Sunstroke time. 


\section{INTRODUCTION}

Des millions d'adultes et d'enfants meurent chaque année dans les pays les plus déminus, en raison de la pollution de l'eau et de l'environnement par des bactéries pathogènes (Tamungang et al., 2016). Selon l'organisation Mondiale de la Santé, 663 millions de personnes au monde n'ont pas accès à l'eau potable (OMS, 2015). Le manque d'eau potable est souvent dû à l'absence d'installation adéquate de traitement de l'eau. Cette situation peut être résolue par la promotion de traitement de l'eau au niveau familial.

L'approche au niveau individuel consiste à faire bouillir de l'eau ou à faire usage des produits chlorés. Mais ces méthodes de désinfection de l'eau posent des problèmes. Bouillir de l'eau exige beaucoup d'énergie que le monde rural trouve dans le bois. Ce type de traitement n'est pas approprié, car il peut conduire à la déforestation. Les méthodes courantes de traitement de l'eau utilisant le chlore et ses dérivés, l'ozone, les lampes ultraviolettes sont coûteuses et souvent inaccessibles aux populations déshéritées.

La désinfection de l'eau par la lumière solaire, une ancienne technique, simple, devrait en principe être une bonne alternative pour la potabilisation de l'eau pour les populations des pays pauvres. L'énergie solaire est universellement présente, durable aussi longtemps qu'il y aura la vie sur terre. La désinfection de l'eau par le soleil implique deux processus: la désinfection par la radiation solaire et le traitement thermal par l'énergie solaire (Sossou et al., 2016). Le meilleur résultat est obtenu pour de petite quantité d'eau de 1 à 21 pour un temps d'exposition de 5 heures à une température de $50{ }^{\circ} \mathrm{C}$ (Conroy et al., 2001). Cette technologie est commercialisée dans les pays pauvres comme la République Démocratique du Congo par SODIS, une entreprise Suisse. Toutefois, l'efficacité de cette technique est souvent mise en doute à cause du manque d'indicateur d'exposition de l'eau au soleil et surtout des variations des conditions climatiques. Cette méthode peut être améliorée par l'usage de l'oxygène singulet, via la photodynamique (Sabbahi et al, 2000 ; Schafer et al., 2000; Sunda et al., 2008; Sunda et al., 2016).

Les effets néfastes des sensibilisateurs de lumière et de l'oxygène sont connus sous le nom de photosensibilisation. L'action photodynamique néfaste et létale sur les micro-organismes tels que les virus, les algues, les champignons, les protozoaires et les animaux est associée à l'oxygène singulet généré dans le milieu (Sunda et al., 2008).

Les photosensibilisateurs sont en fait des molécules endogènes ou exogènes qui sont capables d'absorber la lumière pour produire une réaction chimique qui n'aurait pas eu lieu en leur absence. Les photosensibilisateurs peuvent ou ne pas subir un changement au cours de la réaction. En présence de la lumière solaire, les photosensibilisateurs absorbent de l'énergie qu'ils transfèrent à l'oxygène moléculaire stable (état triple) pour générer l'oxygène singulet.

L'oxygène singulet généré attaque et endommage la plupart des biomolécules et entraîne ainsi la mort cellulaire ou tissulaire. Plusieurs auteurs signalent que les réactions des espèces réactives de l'oxygène "stress oxydatif" interviennent également dans le mode d'action de certaines drogues et médicaments (Tracy et Webster, 2001). Certaines plantes utilisées dans la pharmacopée traditionnelle pour soigner les infections microbiennes et parasitaires sont supposées réagir par un mécanisme de type stress oxydatif (Sunda et al, 2008).

Lors de l'étude de la désinfection de l'eau par photosensibilisation avec les extraits de feuilles de Cassia alata, Cassia occidentalis, Carica papaya, Phyllantus niruri et Coleus kilimandschari, Sunda et al. (2008) ont remarqué que ces extraits avaient un effet photosensibilisateur. L'activité 
photosensibilisatrice trouvée dans ces extraits est due à la présence des molécules photoactives capables de générer l'oxygène singulet (Sunda et al., 2008; Sunda et al., 2009). Malheureusement, l'activité photosensibilisatrice remarquée dans ces plantes n'est accrue que lorsque le milieu est saturé en oxygène (barbotage).

Le présent travail a pour objectif l'étude de la désinfection de l'eau par photosensibilisation avec les extraits flavonoidiques de Aframomum alboviolaceum suite à son utilisation dans la pharmacopée traditionnelle pour le soin d'un certain nombre de maladies par voie photodynamique.

\section{MATERIEL ET METHODES}

Récolte et conditionnement de l'échantillon de plante

Les racines de Aframomum alboviolaceum ont été récoltées dans la commune de Mont Ngafula, au quartier Kimwenza, à Kinshasa. Elles ont été séchées à l'air libre et à l'ombre, puis broyées à l'aide d'un broyeur artisanal jusqu'à l'obtention d'une poudre de $1 \mathrm{~mm}$ de granulométrie.

\section{Prélèvement des échantillons d'eau}

L'eau à analyser provenait d'égouts du Home 10 de l'Université de Kinshasa. Cette eau contenait approximativement $10^{3} \mathrm{UFC} / \mathrm{ml}$ des coliformes fécaux.

\section{Extraction des flavonoïdes}

Les flavonoïdes des racines de Aframomum alboviolaceum ont été extraits avec l'acétate d'éthyle, vue leur solubilité préférentielle dans ce solvant :

- Deux cents grammes $(200 \mathrm{~g}) \mathrm{de}$ poudre de Aframomum alboviolaceum sont macérées dans 2,5 litres de méthanol (deux fois) pendant 48 heures ;

- L'extrait est dissout dans un mélange eau-méthanol : 30 -70;
- Puis soumis à un lavage avec l'éther de pétrole jusqu'à la décoloration complète de la phase éthérée ;

- L'extrait méthanolique est ensuite épuisé avec du chloroforme ;

- On extrait enfin les flavonoïdes de la phase méthanolique avec l'acétate d'éthyle

\section{Chromatographie sur couche mince}

L'extrait d'acétate d'éthyle a été directement soumis la chromatographie sur couche mince (C.C.M.) à l'échelle qualitative pour prédire le nombre de composés contenus dans l'extrait. Deux produits ont été identifiés. Le système d'élution utilisé est le suivant: éther de pétrole-acétate d'éthyleméthanol $(3 / 5 / 1)$.

\section{Chromatographie sur colonne}

Les deux produits identifiés par la chromatographie sur couche mince (C.C.M.) ont été séparés par chromatographie sur colonne avec le gel de silice et le même système d'élution, éther de pétrole-acétate d'éthyle-méthanol : 3/5/1. Ceux-ci ont été par la suite identifiés par la spectroscopie ultraviolette. Les spectres de ces produits sont présentés dans les Figures 1 et 2 . Ces deux flavonoïdes ont été par la suite utilisés dans les tests de photosensibilisation.

\section{Tests de photosensibilisation Préparation des échantillons d'eau}

A $450 \mathrm{ml}$ d'eau distillée, on ajoute 50 $\mathrm{ml}$ d'eau polluée. Par la suite, $10 \mathrm{ml}$ de cette solution est placée dans 10 tubes à essai, puis on y ajoute $0,1 \mathrm{ml}$ d'extrait flavonoïdique. Chaque tube est fermé à l'aide d'un bouchon. Un lot de cinq tubes est exposé au soleil et un autre est gardé à l'obscurité. A chaque heure, les tubes sont retirés dans chaque lot pour les analyses bactériologiques. Le bleu de méthylène, dont l'activité photosensibilisatrice est connue, a été utilisé comme témoin positif. 


\section{Analyses bactériologiques}

Le milieu Rapid'Ecoli a été utilisé pour la culture des coliformes fécaux.

\section{RESULTATS}

\section{Extraction}

L'extraction des flavonoïdes des racines de Aframomum alboviolaceum avec l'acétate d'éthyle a donné un rendement de $2,5 \%$, soit $5 \mathrm{~g}$ d'extraits flavonoïdiques.

\section{Séparation et caractérisation des flavonoïdes}

L'extrait flavonoïdique a été soumis à la chromatographie sur couche mince. Cette analyse a montré deux produits qui ont été par la suite séparés par la chromatographie sur colonne, avec le gel de silice. Les spectres UV de ces deux produits (F1 et F2) sont présentés dans les Figures 1 et 2

L'analyse des spectres (Figures 1 et 2) permet de mettre en évidence les bandes d'absorption caractéristiques des flavonoïdes dérivant des transitions électroniques $\pi-\pi^{*}$ et $n-\pi^{*}$ :
- $\mathrm{F} 1: \lambda_{\max }(\mathrm{nm}): 230$ et 275

- $\mathrm{F} 2: \lambda_{\max }(\mathrm{nm}): 290$

\section{Tests de photosensibilisation}

Sensibilité des coliformes fécaux vis-à-vis des extraits flavonoïdiques

Les résultats des tests de sensibilité des coliformes fécaux vis-à-vis des extraits Flavonoïdiques F1 et F2 sont repris dans les Figures 3 et 4 .

Aucune inhibition significative n'a été notée dans les échantillons d'eau non traités après trois heures d'ensoleillement (Figures 3 et 4). Pour ce qui est des échantillons d'eau traités par les extraits F1 et F2 et gardés à l'obscurité, aucune inhibition n'a été notée du début à la fin des expériences. Par contre, dans ceux traités avec F1 et F2 et exposés au soleil, il a été remarqué une inhibition complète des coliformes fecaux après une heure d'ensoleillement. Ces résultats mettent en évidence l'activité photosensibilisatrice des extraits flavonoidiques F1 et F2.

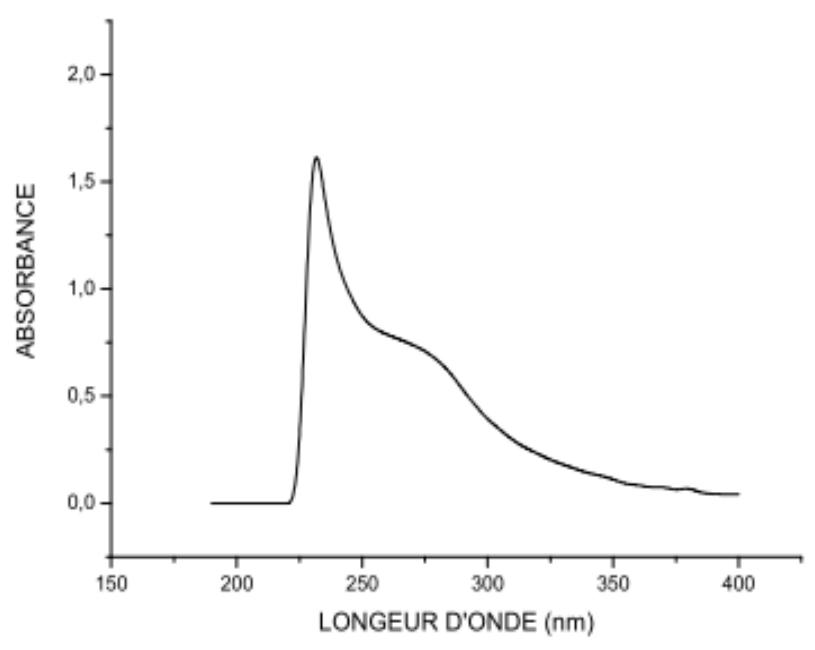

Figure 1 : Spectre UV du produit 1 (F1) dans le méthanol. 


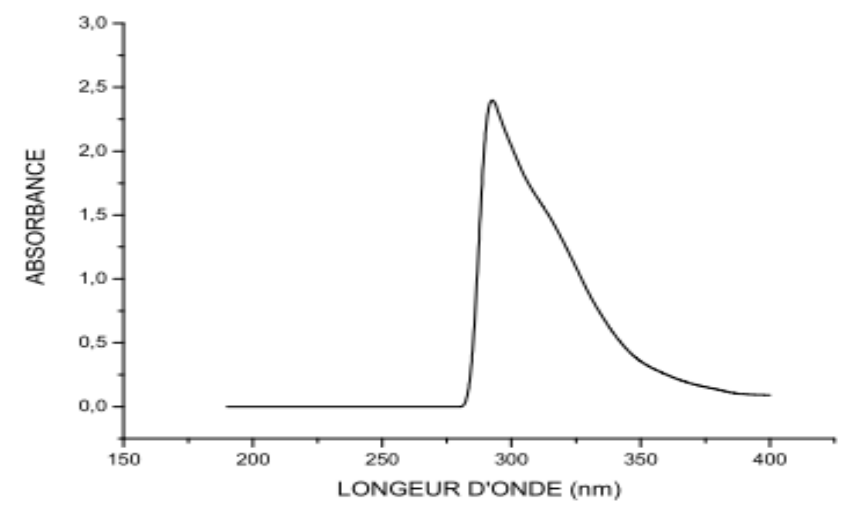

Figure 2 : Spectre UV du produit 2 (F2) dans le méthanol.

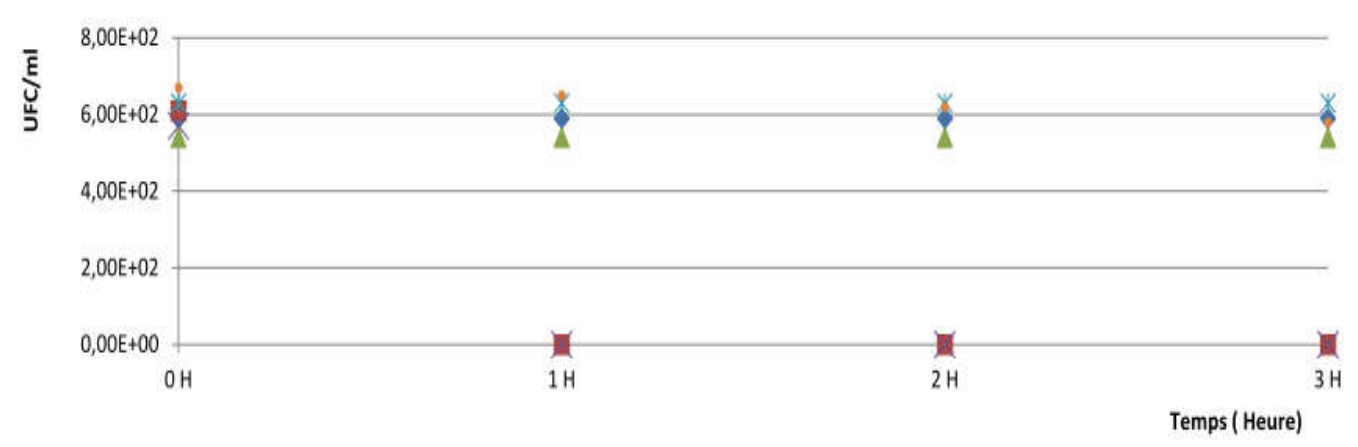

Figure 3 : Abattement des coliformes fécaux avec le temps avec l'extrait F1.

- Eau traitée avec l'extrait F1 et gardée à l'obscurité

$\Delta$ Eau traitée avec le bleu de méthylène et gardée à l'obscurité

* Eau non traitée et gardée à l'obscurité

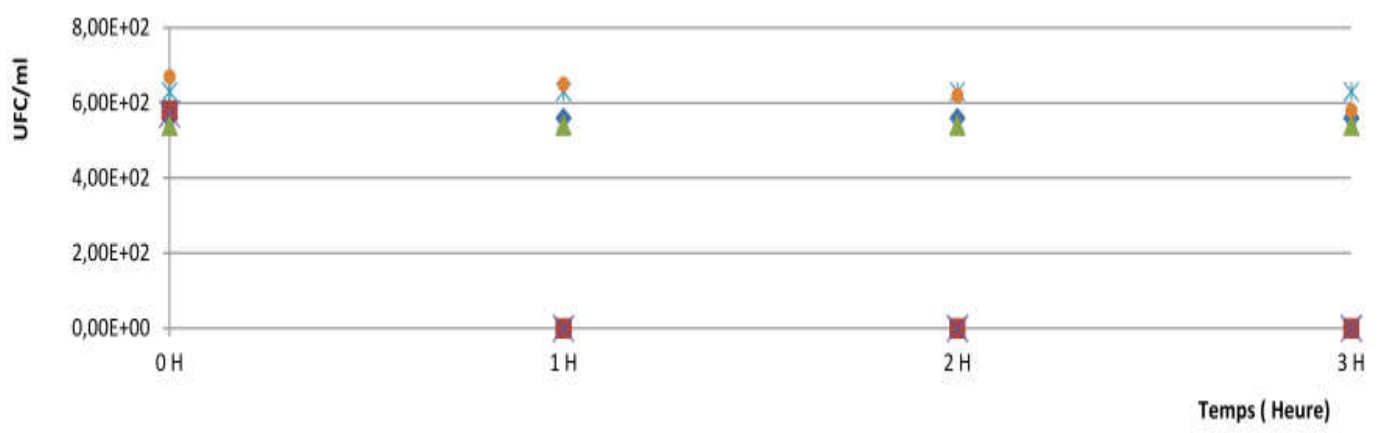

nEau traitée avec l'extrait F1 et exposée au soleil

XEau traitée avec le bleu de méthylène et exposée au soleil

- Eau non traitée et exposée au soleil

Figure 4 : Abattement des coliformes fécaux avec le temps avec l'extrait F2.

- Eau traitée avec l'extrait F2 et gardée à l'obscurité

$\Delta$ Eau traitée avec le bleu de méthylène et gardée à l'obscurité

* Eau non traitée et gardée et à l'obscurité
Eau traitée avec l'extrait $\mathbf{F} 2$ et exposée au soleil

$\times$ Eau traitée avec le bleu de méthylène et exposée au soleil

- Eau non traitée et exposée au soleil 


\section{DISCUSSION Extraction}

Le rendement obtenu est de $2,5 \%$, soit 5 grammes d'extraits flavonoidiques à partir de 200 grammes de poudre sèche. Ce rendement est bon, voire même optimisé, car généralement les métabolites secondaires donnent des rendements inférieurs à $1 \%$.

\section{Séparation et caractérisation des flavonoïdes}

Les deux produits séparés par chromatographie sur colonne à partir de l'extrait d'acétate d'éthyle ont été soumis à la spectroscopie ultraviolette. Les spectres obtenus montrent des bandes d'absorption caractéristiques des Flavonoïdes (Sissa et al., 2010).

\section{Tests de photosensibilisation}

\section{Sensibilité des coliformes fécaux vis-à-vis des extraits flavonoïdiques}

Les résultats obtenus montrent une inhibition complète des coliformes fécaux après une heure d'ensoleillement dans les échantillons d'eau traités par les extraits flavonoidiques F1 et F2. Par contre, dans ceux traités par F1 et F2 et gardés à l'obscurité, il a été observé la présence des coliformes fécaux du début à la fin des expériences. Pour ce qui est des échantillons d'eau non traités et exposés au soleil, il a été noté un abattement négligeable, résultats conformes à ceux obtenus dans plusieurs travaux (Bonnett et al., 2006 ; Sossou et al., 2016; Sunda et al., 2016). Le fait qu'aucune inhibition significative ne soit notée du début à la fin dans les échantillons d'eau non traités après 3 heures d'ensoleillement et qu'une inhibition complète soit notée pour les échantillons d'eau traités et exposés au soleil après une heure d'ensoleillement laisse supposer que l'action conjuguée de lumière et des flavonoïdes (F1 et F2) initie une réaction qui serait responsable de l'inhibition des coliformes. Dans ces conditions les flavonoïdes F1 et F2 se comporteraient comme des molécules photosensibilisatrices. En présence de l'énergie solaire, celles-ci passent de l'état fondamental à l'état excité. En retournant à l'état fondamental, l'énergie emmagasinée est transférée à l'oxygène dissout dans l'eau. L'oxygène dissout dans l'eau passe dès lors de l'état fondamental, triplet, à l'état excité, singulet. Ce dernier provoque la mort des coliformes fécaux présents dans le milieu. C'est ce qui a d'ailleurs été constaté pour le bleu de méthylène, un photosensibilisateur classique qui a été utilisé comme témoin (Figures 3 et 4). Les résultats trouvés dans ce travail sont en accord avec plusieurs travaux réalisés sur la désinfection de l'eau par la photosensibilisation. Tous ces travaux mettent en évidence la génération de l'oxygène singulet par les substances photosensibilisatrices pour la purification de l'eau (Sabbahi et al., 2000; Derosa et al., 2002 ; Emilia et al., 2006 ; Villén L et al., 2006).

\section{Conclusion}

L'étude de la désinfection de l'eau par photosensibilisation avec les extraits flavonoïdiques de Aframomum alboviolaceum montre une inhibition complète des coliformes fécaux présents dans l'eau après une heure d'ensoleillement pour les deux produits (F1 et F2) extraits des racines de cette plante. En ce qui concerne les échantillons d'eau traités avec les extraits flavonoïdiques $\mathrm{F} 1$ et $\mathrm{F} 2$ et gardés à l'obscurité, aucune inhibition n'a été notée du début à la fin des expériences. Ceci laisse supposer que les produits F1 et F2 sont photosensibilisateurs et, qu'en présence du soleil, ceux-ci subissent une photoexcitation qui conduirait à la formation d'oxygène singulet. Ce dernier, une fois généré, détruit 
les coliformes fécaux présents dans l'eau. Ces extraits présentent en outre l'avantage de réagir efficacement sans saturation du milieu en oxygène (barbotage). Des études supplémentaires s'avèrent nécessaires notamment en ce qui concerne la fixation de ces produits sur un support solide tel qu'un polymère ou un verre. La sensibilité des parasites et virus vis-à-vis de ces produits mérite également d'être examinée.

\section{CONFLIT D'INTERETS}

Les auteurs déclarent que le présent article ne fait l'objet d'aucun conflit d'intérêts.

\section{CONTRIBUTIONS DES AUTEURS}

TMS et NKMT sont les instigateurs et PMM a participé à toutes les phases de la recherche.

\section{REFERENCES}

Bonnett R, Krysteve M, Lalov I, Artarsky S. 2006. Water disinfection using photosensitizers immobilized on chitosan. Water Research, 40: 12691275. DOI:10.1016/j.watres.2006.01.014 Conroy R, Elmore-Meegan M, Joyce T, McGuigan K, Barnes J. 2001. Solar disinfection of drinking water protects against cholera in children under 6 years of age. Arch. Dis. Child, 85(4): 293-295. DOI: $10.1136 /$ adc.85.4.293

Derosa M, Crutchley R. 2002. Photosensitized singlet oxygen and its applications. Coordination Chemistry Reviews, 234: 351-371. DOI http://dx.doi.org/10.1016/S00108545(02)00034-6

Emilia M, Jiménez H, Francisco M, David G, Guillermo O. 2006. Solar water disinfection by singlet oxygen photogenerated with polymer-supported $\mathrm{Ru}$ (II) sensitizers. Solar Energy,
80(10): $1382-1387 . \quad$ DOI : http://dx.doi.org/10.1016/j.solener.2005. 04.027

Organisation Mondiale de la Santé (OMS) 2015. Rapport en matière d'assainissement et d'alimentation en eau potable, JMP.

Sabbahi S, Alouini Z, Jemli M. 2000. Etude de la désinfection Photodynamique des eaux usées par le Rose de Bengale sel dissodique RB-2Na. Proc.Int.conf.on waste Water treatment and Reuse Adapted to Mediterranean Area, 137140.

Schafer M, Schmitz C, Facius R, Horneck G, Milow B, Funken KH, Ortner J. 2000. Systematic study of parameters influencing the action of rose Bengal with visible light on bacterial cells: Comparison between the biological effect and singlet oxygen production. Potochem. photobiol., 71(5): 514-523. DOI: $10.1562 / 0031-$ 8655(2000)0710514SSOPIT2.0.CO

Sisa M, Bonnet S, Ferreira D, Westhuizen J. 2010. Photochemistry of Flavonoids. Molecules, 15: 5196-5245. DOI: 10.3390/molecules 15085196

Sossou K, Gbedenu K., Konate Y, Sawadogo B, Ameyapoh Y, Maiga H, Funamizu N. 2006. Damage mechanisms of pathogenic bacteria in drinking water during chlorine and solar disinfection. Int. J. Biol. Chem. Sci., 10(2): 519-532. DOI:

http://dx.doi.org/10.4314/ijbcs.v10i2.6

Sunda M, Rosillon F, Taba KM. 2008. Contribution à l'étude de la désinfection de l'eau par photosensibilisation avec les extraits de plantes. European Journal of Water Quality, 39(2): 199- 209. DOI: http://dx.doi.org/10.1051/water/2008006

Sunda M, Rosillon F, Taba KM, Lami N. 2009. Désinfection de l'eau par 
photosensibilisation avec les huiles essentielles de Citrus Bergamia, Citrus reticulata et Citrus limonum. VIII ${ }^{\text {ième }}$ Congrès International Gruttee, Ressource en Eau: Quels outils Scientifiques pour une gestion durable de leur qualité, Nancy, France, 86 -89.

Sunda M, Taba K.M, Rosillon F, Wathelet B. 2016. Contribution to the study of water disinfection by photosensitization with plant exctracts .C.R. Chimie, 19: 827831.

DOI:

http://dx.doi.org/10.1016/j.crci.2016.01. 012

Tamungang N, Biosengazeh N, Alakeh M, Tameu D. 2006. Contrôle de la qualité des eaux domestiques dans le village Babessi au Nord-Ouest Cameroun. Int. J. Biol. Chem. Sci., 10(3): 1382-1402.
DOI:http://dx.doi.org/10.4314/ijbcs.v10i 3.37

Tracy J, Webster J. 2001. Drugs used in the chemotherapy of protozoal infections: amebiasis, trichomoniasis, trypanosomiasis, leishmaniasis, and other protozoal infections. In Goodman and Gilman's the Pharmacological Basis of Therapeutics $\left(10^{\text {th }}\right.$ ed), Hardman JG, Limbird LE, Gilman AG (eds). McGrawHill: New York.

Villén L, Manjón F, García D, Orellana G. 2006. Solar water disinfection by photocalytic singlet oxygen production in heterogeneous medium. Appl. Catal., 69: 1-9. $\quad$ DOI: http://dx.doi.org/10.1016/j.apcatb.2006.0 5.015 . 